\title{
NORMAL FAMILIES OF HOLOMORPHIC FUNCTIONS
}

\author{
HUAI-HUI CHEN and XIN-HOU HUA
}

(Received 1 October 1992; revised 21 December 1992)

\author{
Communicated by P. C. Fenton
}

\begin{abstract}
Let $a(z)$ be a meromorphic function with only simple poles, and let $k \in \mathbb{N}$. Suppose that $f(z)$ is meromorphic. We first set up an inequality in which $T(r, f)$ is bounded by the counting function of the zeros of $f^{(k)}+a f^{2}$, and then we prove a corresponding normal criterion. An example shows that the restriction on the poles of $a(z)$ is best possible.
\end{abstract}

1991 Mathematics subject classification (Amer. Math. Soc.): 30D45, $30 \mathrm{D} 35$.

\section{Introduction}

One version of the Bloch principle asserts that if we consider a property of meromorphic functions such that every meromorphic function in the plane with that property must be constant, then the class of meromorphic functions in the unit disc with that property uniformly forms a normal family.

Concerning this principle, Drasin [2] proved the following result.

THEOREM A. A family of holomorphic functions is normal if every function in the family satisfies $f^{\prime}-a f^{n} \neq b$, where $a \neq 0$ and $b$ are two constants and $n \geq 3$.

The case $n=2$ was proved by Ye [6].

It is natural to ask whether or not the above results hold if we generalize the constant $a$ to a meromorphic function $a(z)$. If $a(z)$ has no zeros, then the case is easy. The difficult case is when $a(z)$ has zeros and poles.

In this paper, we shall solve this problem. Our main results are the following.

(C) 1995 Australian Mathematical Society 0263-61 15/95 \$A2.00+0.00

Research was supported by the National Natural Science Foundation of China. 
THEOREM 1. Let $f(z)$ be a transcendental entire function, a be a non-zero constant, $k \geq 1$ and $n \geq 2$. Then

$$
T(r, f) \leq(1+2 k) \bar{N}\left(r, 1 /\left(f^{(k)}+a f^{n}\right)\right)+S(r, f) .
$$

THEOREM 2. Let $F$ be a family of holomorphic functions, $a(z)$ and $b(z)$ be meromorphic functions with the property that $a(z)$ has only simple poles and $a(z) \not \equiv 0$, and let $n \geq 2$. If every function $f$ in $F$ satisfies

$$
f^{(k)}+a f^{n} \neq b
$$

then $F$ is a normal family.

We point out that the restriction on the poles of $a(z)$ in Theorem 2 is best possible and necessary. For example, let $F=\left\{f_{n}(z)=n z, n \geq 2\right\}$; then $F$ is not normal at zero. However, $f_{n}^{(k)}+f_{n}^{2} / z^{2} \neq 1$.

REMARK 1. From our proof it is easy to see that Theorem 2 still holds if we replace condition (2) by

$$
f^{(k)}+a_{k-1} f^{(k-1)}+\cdots+a_{1} f^{\prime}+a_{0} f+a f^{n} \neq b,
$$

where $a_{0}, a_{1}, \ldots, a_{k-1}$ are meromorphic functions.

REMARK 2. The special case ' $k=1$ and $a(z)$ and $b(z)$ are holomorphic' was considered by Chen-Fang [1] and Fang-Chen [3]. However, their method does not work for the present cases.

We assume that the reader is familiar with Nevanlinna theory.

\section{Some lemmas}

To prove our results, we need some preliminaries.

LEMMA 1. (HUA [4, THEOREM 2]) Let $f(z)$ be a non-constant meromorphic function, and let $\Psi=f^{n}+P(f)$, where $n \geq 2$ and $P(f)$ is a differential polynomial of $f$ with degree $d(P) \leq n-1$. Then we have at least one of the following:

(i) there exists a small proximity function $c(z)$ (that is, $m(r, c)=S(r, f)$ ) such that $\Psi=\alpha(f+c / n)^{n}$ and

$$
N(r, c) \leq \max \{0, w(P)-n+1\}(\bar{N}(r, 1 / \Psi)+\bar{N}(r, f))+S(r, f),
$$

where $\alpha$ is a non-zero constant and $\alpha=1$ provided that $N(r, f)=S(r, f)$; here $w(P)$ is the weight of $P$; 
(ii) $T(r, f) \leq(1+2 \max \{0, w(P)-n+1\}) \bar{N}(r, 1 / \Psi)$ $+(3+2 \max \{0, w(P)-n+1\}) N(r, f)+S(r, f)$.

LEMMA 2. (XUE-PANG [5]) If the family $F$ of holomorphic functions is not normal at $z_{0}$, then for any positive number $\alpha$, there exist a sequence $z_{n} \rightarrow z_{0}$, a positive sequence $\rho_{n} \rightarrow 0$ and a sequence $\left\{f_{n}\right\} \subset F$ such that $\rho_{n}^{\alpha} f_{n}\left(z_{n}+\rho_{n} \zeta\right)$ is uniformly convergent to a non-constant entire function on any disk $\{|\zeta| \leq R\}$.

LEMMA 3. Suppose that the sequence $\left\{f_{n}\right\}$ of holomorphic functions is uniformly divergent to $\infty$ on the unit disk. Then for any positive integer $k$ and any positive number $\alpha, f_{n}^{(k)} / f_{n}^{1+\alpha}$ is uniformly convergent to 0 on any closed subset of the unit disk.

PROOF. We only need to prove that $f_{n}^{(k)} / f_{n}^{1+\alpha}$ is uniformly convergent to 0 near $z=0$. Without loss of generality, we suppose that $\log \left|f_{n}\right|$ is always non-negative. By applying Poisson's formula to $\log \left|f_{n}\right|$, we obtain

$$
\left|f_{n}(0)\right|^{(1-|z|) /(1+|z|)} \leq\left|f_{n}(z)\right| \leq\left|f_{n}(0)\right|^{(1+|z|) /(1-|z|)} .
$$

Now take a small positive number $r_{0}$ such that

$$
\frac{1+r_{0}}{1-r_{0}} \leq 1+\frac{\alpha}{2}, \quad \frac{1-r_{0}}{1+r_{0}}(1+\alpha) \geq 1+\frac{3}{4} \alpha .
$$

For $|z| \leq r_{0} / 2$, we have

$$
\begin{gathered}
\left|f_{n}^{(k)}(z)\right| \leq \frac{k !}{2 \pi} \int_{|z|=r_{0}} \frac{\left|f_{n}(z)\right|}{\left(r_{0} / 2\right)^{k+1}}|d z| \leq \frac{2^{k+1} k !}{r_{0}^{k}}\left|f_{n}(0)\right|^{1+\alpha / 2}, \\
\frac{\left|f_{n}^{(k)}(z)\right|}{\left|f_{n}(z)\right|^{1+\alpha}} \leq \frac{2^{k+1} k !}{r_{0}^{k}}\left|f_{n}(0)\right|^{-\alpha / 4} .
\end{gathered}
$$

And so, $f_{n}^{(k)} / f_{n}^{1+\alpha}$ is uniformly convergent to 0 . This completes the proof of the lemma.

\section{Proof of theorem 1}

For the sake of simplicity, we only prove the case $n=2$. The case $n>2$ is similar. Put $P(f)=f^{(k)}$ and $\Psi=b P(f)+f^{2}$, where $b=1 / a$. Then $w(P)=k+1$. It is obvious that case (ii) of Lemma 1 is what we need. Thus, by Lemma 1, we only need to consider case (i), that is,

$$
\Psi=(f+c / 2)^{2}
$$


where $c(z)$ is a small proximity function. From (3) and the definition of $\Psi$ we obtain

$$
c^{2}=4 b f^{(k)}-4 c f
$$

From (4) we see that $c$ has no poles. Thus

$$
T(r, c)=S(r, f) .
$$

Let $u=f+c / 2$. Then (3) becomes

$$
\Psi=u^{2} .
$$

Substituting $f=u-c / 2$ into (4) we have

$$
c^{2}-2 b c^{(k)}=4 c u-4 b u^{(k)} .
$$

Now we consider two cases.

Case (a): $c^{2}-2 b c^{(k)} \equiv 0$. If $c$ is not a constant, then $c=2 b c^{(k)} / c$. Thus

$$
T(r, c)=m(r, c)=S(r, c),
$$

which is a contradiction. If $c$ is a constant, then $c \equiv 0$ by the hypothesis of case (a). From this and (4) we deduce that $f(z)$ is a polynomial, a contradiction.

Case (b): $c^{2}-2 b c^{(k)} \not \equiv 0$. From (6) we have

$$
\frac{1}{u}=\frac{4 c}{v}-\frac{4 b}{v} \frac{u^{(k)}}{u}
$$

and so

$$
m(r, 1 / u)=S(r, f)+S(r, u)=S(r, f),
$$

where $v(z)=c^{2}-2 b c^{(k)}$. On the other hand, from (7) we see that any zero of $u$ of order $n>k$ is either a pole of $4 c / v$ with order not less than $n$ or a pole of $4 b / v$ with order $n-k$. Hence, noting (5) and the fact that $v$ and $c$ are small functions of $f$, we have

$$
\begin{aligned}
N(r, 1 / u) & \leq k \bar{N}(r, 1 / u)+N(r, c / v)+N(r, b / v) \\
& \leq k \bar{N}(r, 1 / u)+S(r, f)=k \bar{N}(r, 1 / \Psi)+S(r, f) .
\end{aligned}
$$

Combining this and (8) we obtain

$$
\begin{aligned}
T(r, f) & =T(r, u)+S(r, f)=T(r, 1 / u)+S(r, f) \\
& \leq k \bar{N}(r, 1 / \Psi)+S(r, f) .
\end{aligned}
$$

This completes the proof of Theorem 1 . 


\section{Proof of Theorem 2}

As in the proof of Theorem 1 , we only need to consider the case $n=2$. Let $z_{0}$ be a point which satisfies

$$
a\left(z_{0}\right) \neq 0, \infty, \quad b\left(z_{0}\right) \neq \infty .
$$

If $F$ is not normal at $z_{0}$, then, by Lemma 2 , there exists a sequence

$$
g_{n}(\zeta)=\rho_{n}^{k} f_{n}\left(z_{n}+\rho_{n} \zeta\right)
$$

which is uniformly convergent to a non-constant entire function $g(\zeta)$. We claim that there exists a point $\zeta_{0}$ such that

$$
g^{(k)}\left(\zeta_{0}\right)+a\left(z_{0}\right) g^{2}\left(\zeta_{0}\right)=0
$$

In fact, if $g(\zeta)$ is a non-constant polynomial, then $g^{(k)}(\zeta)+a\left(z_{0}\right) g(\zeta)^{2}$ is also a nonconstant polynomial with degree $2 \operatorname{deg}(g(\zeta))$ by the fact that $a\left(z_{0}\right) \neq 0, \infty$; thus (10) is obvious in this case. If $g(\zeta)$ is transcendental, then (10) follows from Theorem 1 .

Now near $\zeta_{0}$, the function $g^{(k)}(\zeta)+a\left(z_{0}\right) g^{2}(\zeta)$ is the uniform limit of

$$
\begin{aligned}
& g_{n}^{(k)}(\zeta)+a\left(z_{n}+\rho_{n} \zeta\right) g_{n}^{2}(\zeta)-\rho_{n}^{2 k} b\left(z_{n}+\rho_{n} \zeta\right) \\
& \quad=\rho_{n}^{2 k}\left\{f_{n}^{(k)}\left(z_{n}+\rho_{n} \zeta\right)+a\left(z_{n}+\rho_{n} \zeta\right) f_{n}^{2}\left(z_{n}+\rho_{n} \zeta\right)-b\left(z_{n}+\rho_{n} \zeta\right)\right\}
\end{aligned}
$$

By (2) we see that the right-hand side of (11) has no zeros. It follows from (10) and Hurwitz's theorem that

$$
g^{(k)}(\zeta)+a\left(z_{0}\right) g^{2}(\zeta) \equiv 0 .
$$

As in the proof of Theorem 1 , case (a), we deduce that $g(\zeta) \equiv 0$, a contradiction.

Thus, $F$ is normal at the points which satisfy (9).

Now let $z^{\prime}$ be a point which does not satisfy (9). Then there exists a positive number $r_{0}$ such that (9) holds in the set $D=\left\{0<\left|z-z^{\prime}\right|<r_{0}\right\}$. From the above proof we know that $F$ is normal in $D$, so for any sequence in $F$, there exists a subsequence $\left\{f_{n}\right\}$ such that $f_{n}$ is uniformly convergent or uniformly divergent to $\infty$ on any closed subset of $D$. If the first case occurs, then $f_{n}$ is uniformly convergent on any closed subset of $\left\{\left|z-z^{\prime}\right|<r_{0}\right\}$ by the maximum modulus theorem. If the second case occurs, then by Lemma 3,

$$
\left|f_{n}^{(k)}-b\right|<\left|a f_{n}\right|^{2}
$$

holds on $\left|z-z^{\prime}\right|=r_{0} / 2$ for sufficiently large $n$. Now Rouchés theorem asserts that

$$
\begin{aligned}
& n\left(\frac{r_{0}}{2}, z^{\prime}, \varphi_{n}=0\right)-n\left(\frac{r_{0}}{2}, z^{\prime}, \varphi_{n}=\infty\right) \\
& \quad=n\left(\frac{r_{0}}{2}, z^{\prime}, a f^{2}=0\right)-n\left(\frac{r_{0}}{2}, z^{\prime}, a f^{2}=\infty\right),
\end{aligned}
$$


where $\varphi_{n}=f_{n}^{(k)}-b+a f_{n}^{2}$. If $f_{n}$ has zeros in the disk $\left\{\left|z-z^{\prime}\right|<r_{0} / 2\right\}$, then the right hand side of (12) is positive by the assumption that $a(z)$ has at most simple poles. On the other hand, from (2) we see that $n\left(r_{0} / 2, z^{\prime}, \varphi_{n}=0\right)=0$. Thus, the left hand side of (12) cannot be positive. This contradiction shows that $f_{n}$ has no zeros in the set $\left\{\left|z-z^{\prime}\right|<r_{0} / 2\right\}$. By applying the maximum modulus theorem to $1 / f_{n}$ we deduce that $f_{n}$ is uniformly divergent to $\infty$ on $\left\{\left|z-z^{\prime}\right| \leq r_{0} / 2\right\}$. Combining the two cases above, we see that $F$ is normal at $z^{\prime}$.

This completes the proof of Theorem 2 .

\section{Acknowledgement}

We wish to thank the referee for some valuable corrections.

\section{References}

[1] H. H. Chen and M. L. Fang, 'On a theorem of Drasin', Adv. in Math. (China) 20 (1991), 504.

[2] D. Drasin, 'Normal families and the Nevanlinna theory', Acta. Math. 122 (1969), 231-263.

[3] M. L. Fang and H. H. Chen, 'On the normality of a family of holomorphic functions', J. Nanjing Univ. Natural Science 3 (1991), 1-3.

[4] X. H. Hua, 'Some extensions of the Tumura-Clunie theorem', Complex Variables Theory Appl. 16 (1991), 69-77.

[5] G. F. Xue and X. C. Pang, 'Normality criteria of a family of meromorphic function', J. East China Normal Univ. Natur. Sci. Ed. 2 (1988), 15-22.

[6] Y. S. Ye, 'A new normal criterion and its application', Chinese Ann. Math. Ser. A (Supplement) 12 (1991), 44-49.

Department of Mathematics

Nanjing Normal University

Nanjing 210024, P. R. China
Department and Institute of Mathematics

Nanjing University

Nanjing 210008, P. R. China 